\title{
Modeling Developmental Processes Using Latent Growth Structural Equation Methodology
}

\author{
Terry E. Duncan and Susan C. Duncan, Oregon Research Institute \\ Mike Stoolmiller, Oregon Social Learning Center
}

\begin{abstract}
Recent advances in latent growth modeling allow for the testing of complex models regarding developmental trends from both an inter- and intra-individual perspective. The interpretation of model parameters for the latent growth specification is illustrated with a simple two-factor model. An example application of latent growth methodology analyzing developmental change in adolescent alcohol consumption is presented. Findings are discussed with particular reference to the utility of latent growth curve models for assessing developmental processes at both the inter- and intra-individual level across a variety of behavioral domains. Index terms: alcohol consumption, change measurement, developmental models, growth measurement, latent growth models.
\end{abstract}

There has been a recent resurgence of interest in statistical models for time-ordered data using structural equation modeling (SEM) methodology. Interest in models that have the ability to incorporate information concerning the group or population, and also information concerning changes in the individual, has reintroduced the formative work of Rao (1958) and Tucker (1958). The basic notion that Rao and Tucker promoted was the idea that although everyone develops the same way, individual differences are both meaningful and important. These researchers proposed a partial solution to this problem by constructing a procedure that included unspecified longitudinal growth curves. Latent growth models (LGMS) provide a means of modeling individual differences in growth curves.

Although strongly resembling confirmatory factor analysis, the latent growth factors are actually

APPLIED PSYCHOLOGICAL MEASUREMENT

Vol. 18, No. 4, December 1994, pp. 343-354

(C) Copyright 1994 Applied Psychological Measurement Inc. 0146-6216/94/040343-12\$1.85 interpreted as representing individual differences in attributes of growth trajectories over time (McArdle, 1988). For example, two potentially interesting attributes of growth trajectories are rates of change and initial status; in simple, straight-line growth models these are the slope and intercept, respectively. Meredith \& Tisak (1990) noted that repeated measures polynomial analysis of variance models are actually special cases of LGMs in which only the factor means are of interest. In contrast, a fully expanded LGM analysis takes into account both factor means, which correspond to group level information, and variances, which correspond to individual differences. This combination of individual and group level analysis is unique to the procedure. Heuristically, growth curve methodology can be thought of as consisting of two stages. In the first stage, a regression curve, not necessarily linear, is fit to the repeated measures of each individual in the sample. In the second stage, the parameters for an individual's growth curve become the focus of the analysis rather than the original measures.

Thus, the modeling task involves identifying an appropriate growth curve form that will accurately and parsimoniously describe individual development and allow the study of individual differences in the parameters that control the pattern of growth over time. If, for example, the trajectories are well described by a collection of straight lines for a sample of individuals, the developmental model should reflect individual differences in the slopes and intercepts of those lines. Beyond describing and summarizing growth at the group and individual levels, however, the model also can be used to study predictors of individual differences to answer questions about which variables exert important effects 
on the rate of development. Because researchers such as Meredith \& Tisak (1990) and McArdle (1988) have extended the basic model to permit the use of current standards in estimation and testing procedures found in confirmatory SEM programs such as LISREL (Jöreskog \& Sörbom, 1984) and EQS (Bentler, 1989), statistical tests of overall model fit and the significance of individual model parameters are available.

The LGM approach is presented in more technical detail in Meredith \& Tisak (1990), Muthén (1991), and Stoolmiller (1994). Applications of the LGM may be found in Duncan \& Duncan (1994), Duncan, Duncan, \& Hops (1994), Duncan \& Stoolmiller (1993), Stoolmiller, Duncan, Bank, \& Patterson (1993), McArdle (1988), McArdle \& Epstein (1987), and McArdle \& Hamagami (1991). Other approaches to growth modeling can be found in Bryk \& Raudenbush (1987) and Willett, Ayoub, \& Robinson (1991).

Because the LGM uses SEM methodology, it shares many of the same strengths and weaknesses. Some of the strengths of the LGM approach include the capability (1) to test the adequacy of the hypothesized growth form, (2) to incorporate both fixed and time varying covariates, (3) to correct for measurement error in observed indicators, (4) to incorporate growth on several constructs simultaneously, and (5) to develop from the data a common developmental trajectory, thus ruling out cohort effects. Some of the limitations of LGMs include the requirement of large samples, multinormally distributed variables, and the assumption that change is systematically related to the passage of time, at least over the time interval of interest (Burchinal \& Appelbaum, 1991). Evaluating the extent to which a particular LGM is capable of describing the observed pattern of change with respect to time is an important part of growth model testing. In addition, the application of the LGM within the SEM framework depends, at least ideally, on data that are collected when individuals are observed at approximately the same time, and the number and spacing of assessments are the same for all individuals. Longitudinal panel data (Kessler \& Greenberg, 1981) are typical of this design. Ware (1985) referred to these kind of data as "balanced-on-time."

If the number of time points or the spacing between time points vary across individuals, other growth curve techniques are available (e.g., Bryk \& Raudenbush, 1987; Hui \& Berger, 1983; Kleinbaum, 1973). LGMs can be applied to circumstances in which individuals are not measured at the same time intervals; however, specific constraints need to be placed on the models for parameter identification. SEM methodology still can be applied if change on the variables of interest is not related to time. That is, any ordinal variable can be used as the index of the growth function and time is only one useful possibility. However, the models lose their growth curve interpretations. If change is not systematically related to the passage of time, studying individual trajectories over time will not be very informative. In this case, generalized estimating equations methodology (Zeger \& Liang, 1986) is likely to be more appropriate.

Although LGM methodology generally is applied to data generated from a true longitudinal design, given the constraints of time, attrition, and the cost of continual assessment, alternative approaches and methods for accelerating the collection of longitudinal information are often necessary. Bell (1954) advocated the "convergence" method for meeting research needs not satisfied by either longitudinal or cross-sectional methods. This method consists of limited repeated measurements of independent age cohorts resulting in temporally overlapping measurements of the various age groups. This technique, which has gained recent popularity as the "cohort-sequential" design (Nesselroade \& Baltes, 1979), provides a means by which adjacent segments consisting of limited longitudinal data on a specific age cohort can be linked together with similar segments from other temporally related age cohorts to determine the existence of a common developmental trend, or growth curve. In addition, this technique allows the researcher to determine whether those trends observed in the repeated observations are corroborated within short time periods for each age cohort. Within this approach, the researcher approximates a long-term longitudinal 
study by conducting several short-term longitudinal studies of different age cohorts simultaneously.

\section{Development of Adolescent Alcohol Consumption}

Alcohol consumption is considered a relatively universal experience for American adolescents (Sutker, McCleary, \& Allain, 1986). However, few studies have examined the progression or development of alcohol consumption during the time in which this behavior tends to become established (e.g., Duncan et al., 1994; Jessor, 1987; Newcomb \& Bentler, 1986). Given that studies consistently demonstrate the likelihood that alcohol consumption precedes the use of illicit drugs (e.g., Andrews, Hops, Ary, Lichtenstein, \& Tildesley, 1991), longitudinal studies assessing change in adolescent alcohol use and possible correlates of such change are necessary in order to understand future patterns of behavior, including the progression into more serious forms of substance use.

With increased interest in the development of substance use, greater emphasis has been placed on the time dimension and the development of dynamic models pertaining to both intra- and inter-individual development of substance use and its etiology during adolescence. Within this approach, age is viewed as a dimension along which behavior changes are superimposed, forming part of the definition of the dependent variable in developmental studies. This dependent variable is, therefore, defined in terms of specific aspects or parameters of the function describing those changes that occur with age (Wohlwill, 1970).

Extensive longitudinal studies (Patterson, Capaldi, \& Bank, 1990; Patterson, Reid, \& Dishion, 1992; Patterson \& Stouthamer-Loeber, 1984) have suggested that family management practices are precursors to the development of early problem behavior. Although measures of family functioning vary among studies, the bulk of the evidence indicates that negative interactive patterns and parental monitoring both have an independent influence on adolescent problem behavior (Loeber \& Dishion, 1983; Patterson, Reid, \& Dishion, 1992; West \& Farrington, 1973). Poor monitoring, parental permissiveness, and inconsistent limit setting also have been associated with substance use (Coombs \& Landsverk, 1988; Newcomb, Maddahian, Skager, \& Bentler, 1987; Pendergast \& Schaefer, 1974) and antisocial behavior (Canter, 1982; Loeber \& Dishion, 1983). In Patterson et al.'s (1992) longitudinal model of boys' delinquency, poor parental monitoring was consistently associated with boys' antisocial behavior (Patterson \& Bank, 1989). Similarly, adolescent substance use and delinquency are more likely to occur in families in conflict. Increased smoking, drinking, and drug use have shown reliable relationships with coercive adolescent/parent relationships (Coombs \& Landsverk, 1988; Selnow, 1987) and less support and involvement from parents (Coombs \& Landsverk, 1988; Hawkins, Lishner, Catalano, \& Howard, 1986).

In this study, a cohort-sequential model using LGM methodology was tested using EQS (Bentler, 1989). Based on previous findings (Patterson et al., 1992), it was hypothesized that inept monitoring and coercive exchanges would account for individual differences in the growth parameters of adolescent alcohol use over the 5 -year period estimated within the cohort-sequential design.

\section{Method}

\section{Data}

Members of the Kaiser Permanente health maintenance organization (HMO) were recruited to participate in a longitudinal clinical trial to evaluate the efficacy of an adolescent smoking cessation program. In the first phase of recruitment, age eligible adolescents of the HMO were mailed a screening questionnaire that was designed ostensibly to survey young people about their health habits. The questions concerning smoking were used to identify current smokers who could be asked to participate in the second phase of the assessment. In addition, a smaller sample of nonsmokers was recruited to participate in order to better study the correlates of health behavior. For the purpose of the present study, data were assessed from three different age cohorts $(14,15$, and 16 years of age at the initial assessment period), each measured at three occasions over the course of the clinical trial.

These age cohorts were selected so that the time of measurement and age of testing were "approxi- 
mately staggered" (McArdle \& Anderson, 1990). McArdle used this term to mean that the average age of the first cohort at the second measurement period is approximately the average age of the second cohort at the initial measurement, and so on. The sample size was 162 for Cohort 1 (age 14 at initial assessment); 114 for Cohort 2 (age 15 at initial assessment); and 64 for Cohort 3 (age 16 at initial assessment). The complete sample of 340 adolescents (200 males and 140 females) had a mean age of 14.71 years (standard deviation $=.766$ ) at the first assessment, 275 or $81 \%$ were from two-parent families, and the majority of the participants were Caucasian (93.2\%).

Adolescents and their parent(s) completed questionnaires in their homes during a home visit by research staff. Follow-up assessments occurred at approximately 12 and 18 months. Participants were not paid.

\section{Measures}

Table 1 summarizes the items used to assess each of the variables measured.

Alcohol use. Measures of alcohol use were constructed from items assessing adolescents' rates of use during the previous week, month, and 6-month periods. Questions relating to rates of use focused on global frequency of drinks (e.g., "During the last 6 months, how many drinks of alcohol did you have?"). The alcohol use measure represented the adolescent's average use of alcohol during the previous 6-month period. Coefficient alpha ( $\alpha$; Cronbach, 1951) was calculated to determine the internal consistency of the composite measure; values of $.81, .83$, and .83 at Time 1 , Time 2 , and Time 3 , respectively, were obtained.

Monitoring. The index representing inept parental monitoring was defined by six items, four from the parent report (e.g., the degree to which the parent tracks the adolescent's activities outside of school) and two from the adolescent report (e.g., parents allow the adolescent to do things on the weekends without informing the parents of his/her

Table 1

Items Used to Measure Alcohol Use, Monitoring, and Coercion and Their Scaling (For True/False Items: $\mathrm{T}=2$ and $\mathrm{F}=1$; for Likert Items 1-5:1 = Never and 5=Always, Except for $*$ Where $1=$ None and $5=\mathrm{All}$; Items With Ranges of $0-12$ Were Assessed as Frequency of Use: $0,1, \ldots, 10,11=11-20$, and $12=20+$ )

\begin{tabular}{lc}
\hline Scale and Item & Scaling \\
\hline Alcohol Use & $0-12$ \\
How many drinks last week & $0-12$ \\
How many drinks last month & $0-12$ \\
How many drinks last 6 months & $1-5$ \\
Monitoring & $1-5$ \\
My parents let me go anyplace I please without asking & $1-5^{*}$ \\
I get to do things on weekends without telling my parent(s) exactly where I am & $1-5$ \\
How much of your child's time outside of school is unsupervised \\
There usually are no adults around where my child goes after school \\
When your child is at a friend's house, how often is it that the parent(s) or another adult is there \\
When your child attends a party, how often are there supervising adults present & $1-5$ \\
Parent-Child Coercion & $1-5$ \\
Mother-at least 3 times a week we get angry at each other & $\mathrm{TF}$ \\
Father-at least 3 times a wcek we get angry at each other & $\mathrm{TF}$ \\
Mother-we have big arguments over little things & $\mathrm{TF}$ \\
Father-we have big arguments over little things & $\mathrm{TF}$ \\
Mother-at least once a day we get angry at each other & $\mathrm{TF}$ \\
Father-at least once a day we get angry at each other & $\mathrm{TF}$ \\
Mother-in general, I don't think we get along very well & $\mathrm{TF}$ \\
Father-in general, I don't think we get along very well & $\mathrm{TF}$ \\
Mother-family members sometimes hit each other & $\mathrm{TF}$ \\
Father-family members sometimes hit each other & $\mathrm{TF}$ \\
We fight a lot in our family & $1-5$ \\
\hline
\end{tabular}


whereabouts). Items were standardized and summed to create the index of parental monitoring. Items were recoded so that higher scores suggested poorer monitoring on the part of the parents. Internal consistency of the composite measure, $\alpha=.78$, was considered adequate.

Coercion. 11 adolescent report items comprised the measure of adolescent/parent coerciveness [e.g., "We (my mother/father and I) have big arguments about little things." and "We fight a lot in our family."]. With the exception of the latter item, parallel items measured interactions with mother and interactions with father, which were standardized and averaged into a composite parent scale. Items then were summed to create the index of coercion. Higher scores on this scale indicated greater conflict. Internal consistency of the coercion scale was $\alpha=.81$.

\section{The LGM Model}

The model depicted in Figure 1 represents a cohort sequential LGM in which the basic parameters describe a systematic pattern of individual differences in change over time. Figure 1 shows the model for Cohort 1 at ages 14, 15, and 16. For Cohort 2 the same model was used at ages 15-17, and for Cohort 3 at ages 16-18. Thus the model allows for tests of hypotheses concerning convergence across separate groups and the feasibility of specifying a common growth trajectory over the five years represented by the latent variable cohort sequential design. It should be emphasized that equality constraints were imposed for common parameters across the various groups, which, because of the assumption that the hypothesized model is invariant across groups, insures correct estimation of model parameters.

Figare 1

Representation of a Cohort-Sequential LGM Using Three Successive Measurements Across Three Separate Age Cohorts

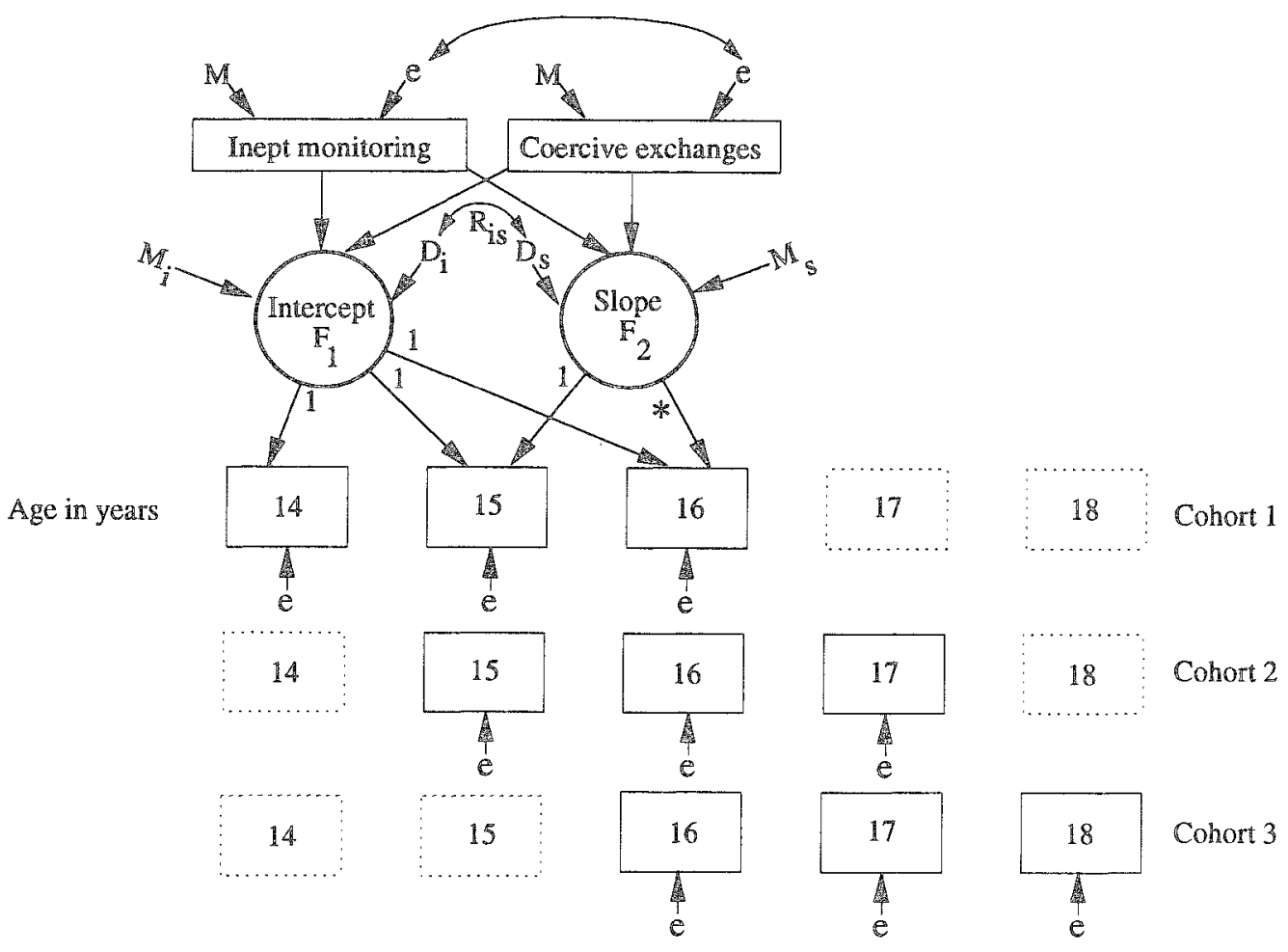

Downloaded from the Digital Conservancy at the University of Minnesota, http://purl.umn.edu/93227. May be reproduced with no cost by students and faculty for academic use. Non-academic reproduction requires payment of royalties through the Copyright Clearance Center, http://www.copyright.com/ 
Because of the overlap between ages in the cohort-sequential design, a longitudinal study spanning ages $14-18$ was created. This method of analysis has been used extensively by McArdle \& Anderson, 1990; McArdle \& Epstein, 1987; and McArdle \& Hamagami, 1991. Because each cohort represents a different pattern of "missingness" in the context of the overall developmental curve, it is possible to build the complete curve using information from all cohorts simultaneously.

The first common factor in Figure 1 is the intercept $\left(F_{1}\right)$ and is a constant for any individual across time. It represents information in the sample concerning the mean, $M_{\mathrm{i}}$, and variance, $D_{\mathrm{i}}$, of the collection of individual intercepts that characterize each individual's growth curve. The $M$ s can be thought of as latent means or as the $\beta$ weights for the regression of the latent factors on a unit constant (a vector of $1 s)$. When the only predictor in a regression equation is the constant 1 , the $\beta$ weight for the regression of the latent factors on the constant is equal to the mean of the dependent variable, called $M$ here.

In standard SEM methodology, the $D_{\mathrm{s}}\left(D_{\mathrm{i}}, D_{\mathrm{s}}\right)$ are usually deviation-from-predicted-value variables, and represent regression residual or error variance. The Ds can have a different interpretation with LGMs. They represent deviation-from-the-mean variables; hence, the $D$ s represent the variance of the latent factors. This is not true in general, however, and when additional predictor variables are in the equation, these parameters have different interpretations. Specifically, the $M$ s now represent the regression intercept, or that part of the dependent variable mean that is not explained by the additional predictor variables, and the Ds are deviation-from-predicted-value variables, generally referred to as disturbance terms, representing unexplained or residual variation. In the model, all measured variables from the three assessment periods have loadings with the common factor representing the intercept, or initial status, constrained at 1.0 .

The second factor, the slope $\left(F_{2}\right)$, represents the shape of an individual's trajectory determined by the repeated measures. The slope factor has a mean, $M_{\mathrm{s}}$, and variance, $D_{\mathrm{s}}$, across the entire sample. $M_{\mathrm{s}}$ and $D_{\mathrm{s}}$ can be estimated from the observed data $\left(M_{\mathrm{i}}\right.$ and $D_{\mathrm{i}}$ the mean and variance, respectively, associated with the intercept also can be estimated from the observed data). The nonconstrained parameter estimates for the measured variables form the basic shape of the reference curve. $F_{1}$ and $F_{2}$ are allowed to covary, $R_{\text {is }}$ which is represented by the double-headed arrow between the two factors. The es correspond to timespecific errors in the observed variables.

The scaling of the slope can be controlled by the choice of loadings on $F_{2}$. Rather than using a model with fixed parameter restrictions (e.g., straight-line growth), a developmental function was specified that reflected an optimal patterning over occasions for the changes in alcohol use scores. Within this approach, the freely estimated parameters reflect the developmental function with maximal fit to the data (Rao, 1958). In this example, the loadings of the measured variables from the Time 1 and Time 2 assessments with the common factor representing the slope of the reference curve, were constrained at values of 0 and 1.0 , respectively. Although the choice of loadings was somewhat arbitrary, it is important to note that $F_{1}$ is inextricably bound to the time scale. By shifting the factor loadings on $F_{2}$, the scale of time is altered and this in turn affects the meaning and interpretation of $M_{\mathrm{i}}$ and $D_{\mathrm{i}}$. To illustrate this idea consider the collection of straight lines depicted in Figure 2.

Notice that with movement from right to left along the horizontal time axis, the variance at any given time point changes. The rank order on $V$, some variable of interest, from highest to lowest also changes. If the factor loadings were selected to force $F_{1}$ to be located at Time 0 , the variance of $F_{1}$ would be different than if it were located at Time 1. Similarly, the correlation and the covariance between $F_{1}$ and $F_{2}$ will vary depending on how the factor loadings are selected.

Changing the fixed loadings, which changes the time scale, simply rescales $M_{\mathrm{s}}$ and $D_{\mathrm{s}}$, in this case by constants; however, this is not true for $M_{\mathrm{i}}$ and $D_{i}$. Rescaling by constants does not change the fundamental meaning or affect significance tests of the parameters. It also will not affect the level of correlation of $F_{2}$ with other predictors in the model. Fix- 
Figne 2

Straight Line Growth Curves

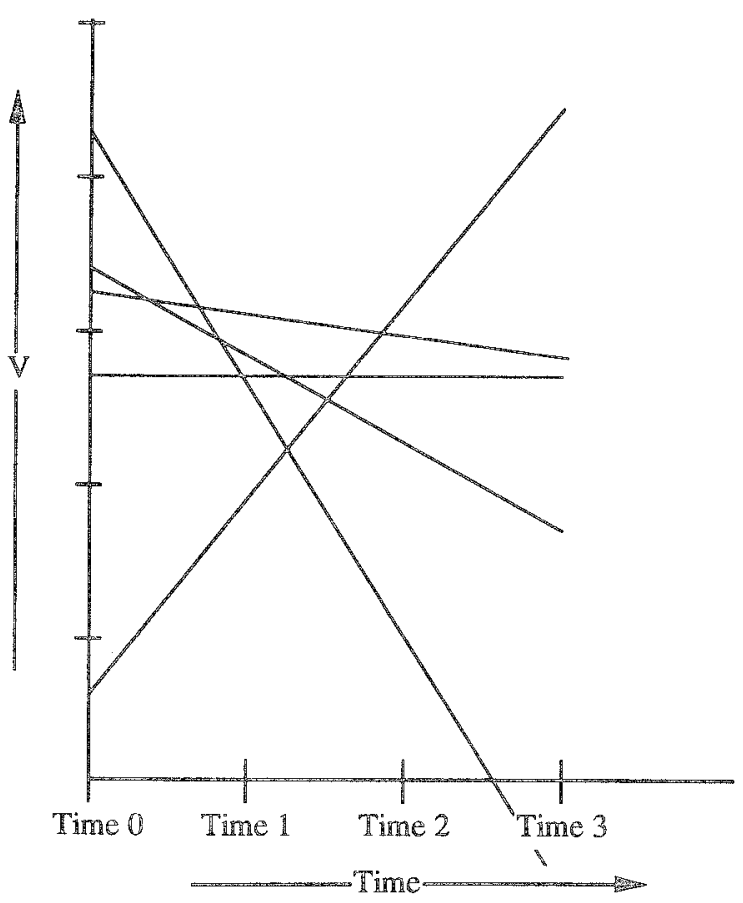

ing the parameter estimates at these values, however, scales the metric for growth rate allowing for an unambiguous interpretation of $F_{1}$ as the initial developmental status that has been corrected for measurement error.

To further explain the nature of the LGM, consider the equation for a growth curve for a single individual with three data points:

$v_{j}=b_{1}+t_{j} b_{2}+e_{j}$

where

$b_{1}$ is the intercept,

$b_{2}$ is the slope (amount of vertical increase per unit of horizontal run of the growth curve),

$t_{j}$ is the $j$ th value of time, and

$e_{j}$ represents the time-specific errors of prediction.

For this hypothetical individual, therefore, the set of equations is:

$v_{1}=b_{1}+0 b_{2}+e_{1}$

$v_{2}=b_{1}+1 b_{2}+e_{2}$, and

$v_{3}=b_{1}+\lambda b_{2}+e_{3}$.

Relating these equations to Figure 1 , for any given individual, $b$, corresponds to the intercept factor score $\left(F_{1}\right), b_{2}$ corresponds to the slope factor score $\left(F_{2}\right), t_{j}$ is the factor loading for $F_{2}$ where the $\lambda$ in Equation 4 represents factor loadings to be estimated, and $e_{j}$ corresponds to the time-specific errors of prediction.

EQS 3.0 (Bentler, 1989) was used to test the hy= pothesized cohor-sequential growth model (complete program specifications and input data are available from the authors). In this analysis, the number of equations equals the number of dependent variables-variables that have unidirectional arrows directed toward them in Figure 1. Therefore, there was an equation for each observed variable, $V$, and the latent or unobserved variables, $F_{1}$ and $F_{2}$. All other variables not having a unidirectional arrow directed toward them-the es, representing errors in measurement, and $D_{\mathrm{S}}$, disturbances in the latent variableswere independent variables; they did not have equations but instead had variances and covariances as parameters. Each measured dependent variable, $V$, was a linear combination of both common factors and one independent error variable.

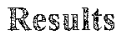

A common approach to SEM has been to test the underlying structure of an hypothesized model and to report some index of the goodness of fit of that model to the data. A number of methods exist that allow determination of the degree of data fit to the hypothesized model and assessment of whether the fit could be improved as a function of testing alternative models. Commonly accepted indices of fit used to evaluate model fit are the $\chi^{2}$ test statistic and various goodness-of-fit indexes. Three goodness-of-fit indexes-NFI, NNFI, and CFI-based on the $\chi^{2}$ test statistic and the mull model of uncorrelated or independent variables are provided by EQS. The Bentler-Bonnet (1980) nomed fir index (NFI) is computed as

$\mathbb{N F}=1-\frac{\chi_{k}^{2}}{x_{i}^{2}}$, 
where $\chi_{k}^{2}$ and $\chi_{i}^{2}$ are the $\chi^{2}$ values obtained for the model of interest and the corresponding independence model, respectively. Values of NFI range between 0 and 1 , with values greater than .9 desirable.

A non-normed fit index (NNFI) takes into account the degrees of freedom $(d f)$ of the model and is computed as

$$
\mathrm{NNFI}=\frac{\left(f_{i}-f_{k}\right)}{\left(f_{i}-1\right)},
$$

where $f_{i}=n \chi_{i}^{2} / d f_{i}$ and $f_{k}=n \chi_{k}^{2} / d f_{k}$ are $\chi^{2}$ variates divided by the associated $d f$. NNFI can exceed NFI in value and can be outside the $0-1$ range.

The comparative fit index (CFI; Bentler, 1990) is computed as

$\mathrm{CFI}=1-\frac{\tau_{k}}{\tau_{i}}$,

where $\tau_{k}=\max \left[\left(n \chi_{k}^{2}-d f_{k}\right), 0\right]$ based on the model of interest, and $\tau_{i}=\max \left[\left(n \chi_{i}^{2}-d f_{i}\right),\left(n \chi_{k}^{2}-d f_{k}\right), 0\right]$. Both the NNFI and CFI reflect model fit well at all sample sizes.

Descriptive statistics for the various age cohorts are presented in Table 2. Univariate values of skewness and kurtosis were in most cases minimal, which indicates that the assumption of approximate normality was tenable. Approximate normality justifies the use of normal theory maximum likelihood estimation techniques found in SEM programs such as LISREL (Jöreskog \& Sörbom, 1984) and EQS (Bentler, 1989).

Fitting the LGM to the alcohol use data resulted in a mean intercept value of $M_{\mathrm{i}}=5.885, t=9.048, p<$ .001 (testing whether the parameter was significantly different from 0.0), and mean slope of $M_{\mathrm{s}}=3.516, t=$ $5.401, p<.001$. The intercept variance was $D_{\mathrm{i}}=$ $45.787, t=5.095, p<.001$, and the variance of the latent slope scores was $D_{\mathrm{s}}=18.924, t=2.296, p<$ .01 , which indicates that substantial variation existed in individual differences regarding initial status and the trajectories of alcohol use. The estimated correlation between initial status and slope scores (rate of change or trajectory) was $R_{\mathrm{is}}=-.335, t=-1.405, p<$ .01 . The variance of the errors in the measured variables, $E=33.555, t=15.574, p<.001$, was required to be equal across groups. The growth curve param-
Talde 2

Descriptive Statistics for the Alcohol Use and Social Context Variables

\begin{tabular}{lrrrr}
\hline $\begin{array}{l}\text { Cohort and } \\
\text { Variable }\end{array}$ & Mean & $\begin{array}{c}\text { Standard } \\
\text { Deviation }\end{array}$ & $\begin{array}{c}\text { Skew- } \\
\text { ness }\end{array}$ & $\begin{array}{r}\text { Kurto- } \\
\text { sis }\end{array}$ \\
\hline $\begin{array}{l}\text { Cohort 1 } \\
\text { Alcohol Use }\end{array}$ & & & & \\
$\quad$ Time 1 & 6.17 & 8.91 & 1.10 & -.06 \\
$\quad$ Time 2 & 10.03 & 8.84 & .28 & -1.58 \\
$\quad$ Time 3 & 9.34 & 9.04 & .28 & -1.54 \\
Monitoring & .28 & .53 & 1.74 & -1.06 \\
Coerciveness & .14 & .80 & -1.29 & .15 \\
Cohort 2 & & & & \\
Alcohol Use & & & & \\
Time 1 & 8.27 & 8.48 & .67 & -1.10 \\
$\quad$ Time 2 & 10.05 & 8.98 & .22 & -1.60 \\
$\quad$ Time 3 & 10.04 & 9.12 & .15 & -1.65 \\
Monitoring & .19 & .54 & -1.08 & -.57 \\
Coerciveness & .14 & .73 & -1.03 & -.02 \\
Cohort 3 & & & & \\
Alcohol Use & & & & \\
Time 1 & 8.98 & 9.43 & .44 & -1.24 \\
$\quad$ Time 2 & 9.77 & 9.02 & .23 & -1.60 \\
$\quad$ Time 3 & 11.61 & 8.99 & -.14 & -1.64 \\
Monitoring & .10 & .56 & -.68 & .63 \\
Coerciveness & .02 & .69 & .25 & -1.00 \\
\hline
\end{tabular}

eters (or basis terms corresponding to the $\lambda$ s in Equations 2-4) of $0,1,1.028,1.213$, and 1.549 for alcohol use at $t_{1}$ through $t_{5}$ respectively, revealed evidence of a general upward development in the use of alcohol over the 5-year period covered by the cohortsequential design. These parameter estimates are directly proportional to the estimated means (the observed variable means corrected for measurement error), $5.885,9.401,9.501,10.151,11.333$, over the same period. Reported values for the fit indexes$\mathrm{NFI}=950, \mathrm{NNFI}=1.006, \mathrm{CFI}=1.000, \chi^{2}=14.637$ with $18 d f, p=.686$-indicated an adequate fit of the model to the data. Figure 3 provides a graphical representation of the adequacy of the model in estimating the common developmental trajectory. The figure shows the expected latent curve developed from the estimated means, in relation to the staggered age group curves developed from the observed alcohol use means, for each age cohort.

Fitting a simple linear LGM resulted in the linear slope factor variance being constrained at 0 for these data. Incorrect $d f$ for the overall model fit $\left(\chi^{2}=36.433\right.$ with $\left.20 d f, p=.012\right)$, resulted from 
Fighe 3

Staggered Age Group Means of Adolescent Alcohol Use

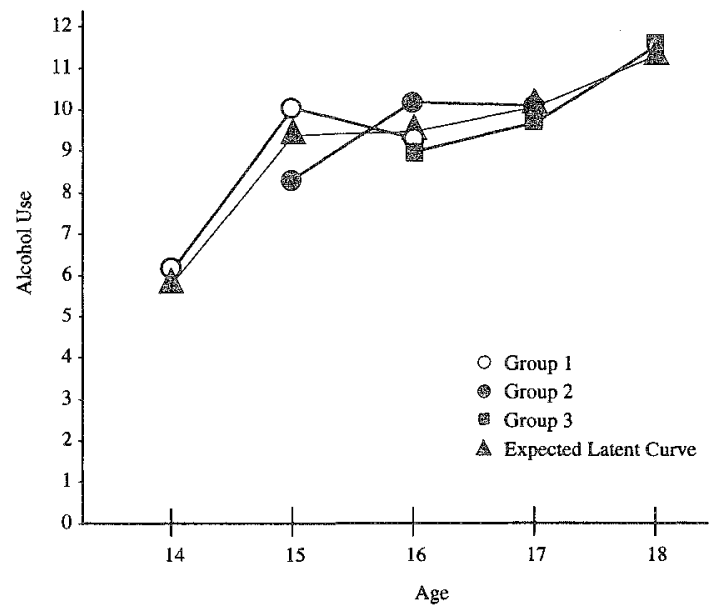

a linear dependency of one equality constraint and the return of $1 d f$ to the model. Although relaxing specific equality constraints would have allowed for model convergence without the lower bound constraint of the slope factor variance being imposed by EQS, this solution was considered inappropriate.

Having adequately modeled a common trajectory, Figure 1 also specifies two covariates, inept monitoring and coercive adolescent/parent exchanges, thought to have significant impact on growth parameters representing individual differences in both initial status and change over time (intercept and slope, respectively). Model-fitting procedures for the tests of convergence (i.e., specifying that the effects of the covariates on the developmental parameters were the same across the various age cohorts), yielded $\chi^{2}=31.369$ with 40 $d f(p=.833)$ and fit indexes of NFI $=.911, \mathrm{NNFI}=$ 1.020 , and $\mathrm{CFI}=1.000$. Estimated regression parameters from the converged model suggested that coercive adolescent/parent exchanges functioned to increase initial levels (or the intercept) of alcohol use $(\beta=.245, t=2.469, p<.05)$, but had no effect on changes in alcohol use (the slope). In contrast, inept monitoring was suggestive of higher rates of change $(\beta=.246, t=1.791, p<.08)$, but had no effect on initial status.

\section{Discussion}

Modeling growth or developmental process within the latent variable SEM format is a potentially valuable methodology that many researchers feel is, unfortunately, underused (see, e.g., Bryk \& Raudenbush, 1987; Meredith \& Tisak, 1990; Rogosa, Brandt, \& Zimowski, 1982; Rogosa \& Willett, 1985; Willett et al., 1991, for comprehensive expositions on the benefits of growth modeling techniques). One purpose of the present study, therefore, was to demonstrate the utility of growth modeling methodology by investigating developmental change in adolescent alcohol use and to examine parental monitoring and coercive exchanges as possible correlates of such change.

The general two-factor LGM approach has many advantages that make it useful in the testing and evaluation of developmental models. With the judicious choice of factor loadings to identify the model, the intercept and slope factors have straightforward interpretations as initial status and change, respectively. Using this parameterization, investigators can study predictors of change separately from correlates of initial status. Although it is possible to keep adding factors until a satisfactory fit to the data is obtained, LGM methodology is most powerful if a small number of factors can adequately describe the data. The characteristics of the collection of developmental trajectories that comprise the sample not only determine the magnitudes of the estimated model parameters, but the number of factors that will be adequate to describe the data.

Results of the present study indicated a good fit for the hypothesized model that specified mean level increases in alcohol use over time. This supports other studies that have found a general increase in the use of alcohol during adolescence (e.g., Duncan \& Duncan, 1994; Duncan et al., 1994). Because of the overlap between ages in the cohort-sequential design, statistical tests [in the form of Lagrange multipliers (Satorra, 1989)] of whether these separate curves converged on a single developmental curve were conducted. On the basis of these findings, it appears that a complete longitudinal curve spanning a five-year period can be esti- 
mated from three age cohorts using only two years (three assessments) of data. The provision of an estimate of long-term developmental trajectories with minimal longitudinal data represents an efficient method for analyzing development.

Although SEM has become a useful methodology for testing complex models representing developmental processes, proponents of LGM methodology have pointed to some potentially serious shortcomings inherent in typically used developmental models (i.e., linear panel models) that incorporate autoregressive effects (Kessler \& Greenberg, 1981). Specifically, serial correlations of errors lead to a correlation between lagged endogenous variables and the prediction errors for the unlagged endogenous variable. When this occurs, none of the structural coefficients can be estimated without bias. However, if the form of the serial correlation is assumed to be $n$ th-order autoregressive, it is possible to transform the structural equation so that the correlation between the prediction error and the lagged endogenous variable disappears. Once this is done, it is possible to estimate the transformed equation without bias. The order of the autoregressive effect represents the number of lagged time units for which errors are serially correlated.

Rogosa (1988) demonstrated the hazards involved in using linear panel models to study the amount of change between two time points when nonlinear growth processes are operative. Linear panel models are not optimal for studying linear growth processes either. All else being equal, the precision of a slope estimate in a linear growth model is an increasing function of the number of time points employed, but it takes at least three points to even begin to evaluate the precision of the estimate. In essence, if it is believed that change is systematically related to the passage of time (i.e., growth processes are operative) then growth models should be employed. If the developmental process of interest is not agedependent, then linear panel models can be useful.

With the development of SEM, researchers now have a powerful tool for the construction, estimation, and testing of complex models involving developmental processes across a plethora of behavioral domains. However, theory testing within the SEM paradigm requires that the hypotheses be clearly specified and that adequate measurement strategies be in place. With each test of the hypothesized model, relationships can be examined between the theoretical constructs of interest, enabling the researcher to discard some hypotheses and to clarify others. Those hypotheses with promise can be used in more rigorous tests, such as experimental manipulation of the variables thought to have causal relationships. Naturally, there is no single statistical procedure for the analysis of longitudinal data, because different research questions dictate different data structures and, subsequently, different statistical models and methods. However, the use of growth curve methodology, in any form, will likely bolster researchers' success in modeling development at both the inter- and intra-individual levels, and in identifying important predictors of such change.

\section{References}

Andrews, J. A., Hops, H., Ary, D., Lichtenstein, E., \& Tildesley, E. (1991). The construction, validation and use of a Guttman scale of adolescent substance use: An investigation of family relationships. The Journal of Drug Issues, 21, 557-572.

Bell, R. Q. (1954). An experimental test of the accelerated longitudinal approach. Child Development, 25 , 281-286.

Bentler, P. M. (1989). EQS structural equations program manual. Los Angeles: BMDP Statistical Software, Inc.

Bentler, P. M. (1990). Comparative fit indices in structural models. Psychological Bulletin, 107, 238-246.

Bentler, P. M., \& Bonett, D. G. (1980). Significance tests and goodness of fit in covariance structures. Psychological Bulletin, 88, 588-606.

Bryk, A. S., \& Raudenbush, S. W. (1987). Application of hierarchical linear models to assessing change. Psychological Bulletin, 101, 147-158.

Burchinal, M., \& Appelbaum, M. I. (1991). Estimating individual developmental functions: Methods and their assumptions. Child Development, 62, 23-43.

Canter, R. J. (1982). Family correlates of male and female delinquency. Criminology, 20, 149-167.

Coombs, R. H., \& Landsverk, J. (1988). Parenting styles and substance use during childhood and adolescence. Joumal of Marriage and the Family, 50, 473-482.

Duncan, S. C., \& Duncan, T. E. (1994). Modeling incomplete longitudinal substance use data using latent variable growth curve methodology. Multivariate Behavioral Research, 29, 313-338. 
Duncan, T. E., Duncan, S. C., \& Hops, H. (1994). The effects of family cohesiveness and peer encouragement on the development of adolescent alcohol use: A cohort-sequential approach to the analysis of longitudinal data. Journal of Studies on Alcohol, 55, 588-599.

Duncan, T. E., \& Stoolmiller, M. (1993). Modeling social and psychological determinants of exercise behaviors via structural equation systems. Research Quarterly for Exercise and Sport, 64, 1-16.

Gollob, H. F., \& Reichardt, C. S. (1987). Taking account of time lags in causal models. Child Development, $58,80-92$.

Hawkins, J. D., Lishner, D. M., Catalano, R. F., \& Howard, M. O. (1986). Childhood predictors and the prevention of adolescent substance abuse: Toward an empirically grounded theory. Journal of Children in Contemporary Society, 18, 11-48.

Hui, S. L., \& Berger, J. O. (1983). Empirical Bayes estimation of rates in longitudinal studies. Journal of the American Statistical Association, 78, 753-760.

Jessor, R. (1987). Problem-behavior theory, psychosocial development, and adolescent problem drinking. British Journal of Addiction, 82, 331-342.

Jöreskog, K. G., \& Sörbom, D. (1984). LISREL VI: Analysis of linear structural relationships by the method of maximum likelihood. Chicago: Scientific Software.

Kessler, R. C., \& Greenberg, D. F. (1981). Linear panel analysis: Models of quantitative change. New York: Academic Press.

Kleinbaum, D. C. (1973). A generalization of the growth curve model which allows missing data. Journal of Multivariate Analysis, 3, 117-124.

Loeber, R., \& Dishion, T. J. (1983). Early predictors of male delinquency: A review. Psychological Bulletin, 94, 68-99.

McArdle, J. J. (1988). Dynamic but structural equation modeling of repeated measures data. In $R$. B. Cattell \& J. Nesselroade (Eds.), Handbook of multivariate experimental psychology (2nd ed.; pp. 561-614). New York: Plenum Press.

McArdle, J. J., \& Anderson, E. R. (1990). Latent growth models for research on aging. In L. E. Biren \& K.W. Schaie (Eds.), The handbook of psychology of aging (3rd ed.; pp. 21-44). San Diego: Academic Press.

McArdle, J. J., \& Epstein, D. (1987). Latent growth curves within developmental structural equation models. Child Development, 58, 110-133.

McArdle, J. J., \& Hamagami, F. (1991). Modeling incomplete longitudinal and cross-sectional data using latent growth struetural models. In L. M. Collins \& J. C. Horn (Eds.), Best methods for the analysis of change (pp. 276-304). Washington DC: American Psychological Association.

Meredith, W., \& Tisak, J. (1990). Latent curve analysis.
Psychometrika, 55, 107-122.

Muthén, B. O. (1991). Analysis of longitudinal data using latent variable models with varying parameters. In L. C. Collins \& J. L. Horn (Eds.), Best methods for the analysis of change (pp. 1-17). Washington DC: American Psychological Association.

Nesselroade, J. R., \& Baltes, P. B. (1979). Longitudinal research in the study of behavior and development. New York: Academic Press.

Newcomb, M. D., \& Bentler, P. M. (1986). Drug use, educational aspirations, and work force involvement: The transition from adolescence to young adulthood. American Journal of Community Psychology, 14, 303-321.

Newcomb, M. D., Maddahian, E., Skager, R., \& Bentler, P. M. (1987). Substance abuse and psychosocial risk factors among teenagers: Associations with sex, age, ethnicity, and type of school. American Journal of Drug and Alcohol Abuse, 13, 413-433.

Patterson, G. R., \& Bank, L. (1989). Some amplifying mechanisms for pathologic processes in families. In M. R. Gunnar \& E. Thelen (Eds.), Systems and development: The Minnesota symposium on child psychology (Vol. 22; pp. 167-209). Hillsdale NJ: Erlbaum.

Patterson, G. R., Capaldi, D., \& Bank, L. (1990). An early starter model for predicting delinquency. In $D$. Pepler \& K. H. Rubin (Eds.), The development and treatment of childhood aggression (pp. 247-279). Hillsdale NJ: Erlbaum.

Patterson, G. R., Reid, J. B., \& Dishion, T. J. (1992). Antisocial boys. Eugene OR: Castalia.

Patterson, G. R., \& Stouthamer-Loeber, M. (1984). The correlation of family management practices and delinquency. Child Development, 55, 1299-1307.

Pendergast, T. J., \& Schaefer, E. D. (1974). Correlates of drinking and drunkenness among school students. Quarterly Journal of Studies on Alcohol, 35, 232-242.

Rao, C. R. (1958). Some statistical methods for comparison of growth curves. Biometrics, 14, 1-17.

Rogosa, D. R. (1988). Myths about longitudinal research. In K. W. Schaie, R. T. Campbell, W. M. Meredith, \& S. C. Rawlings (Eds.), Methodological issues in aging research (pp, 171-209). New York: Springer.

Rogosa, D. R., Brandt, D., \& Zimowski, M. (1982). A growth curve approach to the measure of change. Psychological Bulletin, 92, 726-748.

Rogosa, D. R, \& Willett, J. B. (1985). Understanding correlates of change by modeling individual differences in growth. Psychometrika, 50, 203-228.

Satorra, A. (1989). Altemative test criteria in covariance structure analysis: A unified approach. Psychometrika, $54,131-151$.

Selnow, G. W. (1987). Parent-child relationships and single and two parent families: Implications for substance usage. Joumal of Drug Education, 17, 315-326. 
Stoolmiller, M. (1994). Antisocial behavior, delinquent peer association and unsupervised wandering for boys: Growth and change from childhood to early adolescence. Multivariate Behavioral Research, 29, 263-288.

Stoolmiller, M., Duncan, T. E., Bank, L., \& Patterson, G. R. (1993). Some problems and solutions in the study of change: Significant patterns in client resistance. Journal of Clinical and Consulting Psychology, 61, 920-928.

Sutker, P. B., McCleary, G. E., \& Allain, A. N. (1986). Adolescent alcohol abuse. In R. A. Feldman \& A. R. Streffman (Eds.), Advances in adolescent mental health (pp. 195-253). Greenwich CT: JAI Press.

Tucker, L. R. (1958). Determination of parameters of a functional relation by factor analysis. Psychometrika, $23,19-23$.

Ware, J. H. (1985). Linear models for the analysis of longitudinal studies. American Statistician, 39, 95-101.

West, D. J., \& Farrington, D. T. (1973). Who becomes delinquent? London: Heinemann Educational Books, Ltd.

Willett, J. B., Ayoub, C. C., \& Robinson, D. (1991). Using growth modeling to examine systematic differ- ences in growth: An example of change in the function of families at risk of maladaptive parenting, child abuse, or neglect. Journal of Consulting and Clinical Psychology, 59, 38-47.

Wohlwill, J. F. (1970). The age variable in psychological research. Psychological Review, 77, 49-64.

Zeger, S. L., \& Liang, K. Y. (1986). Longitudinal data analysis for discrete and continuous outcomes. Biometrics, 42, 121-130.

\section{Acknowledgments}

Data were collected under the auspices of Grant No. CA 44648 from the National Cancer Institute, U.S. PHS. Preparation of this article was supported in part by Grant No. MH 46690 from the Prevention Research Branch, NIMH, U.S. PHS; Grant No. CA 38273 from the National Cancer Institute, U.S. PHS; and Grants No. DA 07306 and DA 07389 from the National Institute on Drug Abuse, U. S. PHS.

\section{Author's Address}

Send requests for reprints or further information to Terry E. Duncan, Oregon Research Institute, 1715 Franklin Blvd., Eugene OR 97403, U.S.A. 